\title{
Flow Analysis and Optimization of Supersonic Rocket Engine Nozzle at Various Divergent Angle using Computational Fluid Dynamics (CFD)
}

\author{
Karna S. Patel \\ (B.Tech, U.V. Patel college of Engineering, Ganpat University)
}

\begin{abstract}
CFD is a branch of Fluid Mechanics which rely on numerical methods and algorithms to solve and analyze problem that involves fluid flow. CFD analysis has been conducted to analyze flow pattern of supersonic rocket nozzle at various degree of divergent angle by using two dimensional axis-symmetric model, which solves governing equation by a control volume method. Variation in parameters like velocity, static pressure, turbulence intensity and temperature are being analyzed. Objective of this research is to is to investigate best suited divergent angle. The phenomena of oblique shock is visualized and it was found that at 15 degree of divergent angle it is completely eliminated from nozzle. Also intensity of velocity is found to have an increasing trend with increment in divergent angle thereby obtaining an optimum divergent angle which would eliminates instabilities due to shock and satisfy the thrust requirement from the nozzle.
\end{abstract}

Keywords: shockwave; divergent angle; CFD analysis.

\section{Introduction}

Nozzle is used to convert the chemical-thermal energy generated in the combustion chamber into kinetic energy. The nozzle converts the low velocity, high pressure, high temperature gas in the combustion chamber into high velocity gas of lower pressure and temperature. Swedish engineer of French descent who, in trying to develop a more efficient steam engine, designed a turbine that was turned by jets of steam. The critical component - the one in which heat energy of the hot high-pressure steam from the boiler was converted into kinetic energy - was the nozzle from which the jet blew onto the wheel. De Laval found that the most efficient conversion occurred when the nozzle first narrowed, increasing the speed of the jet to the speed of sound, and then expanded again. Above the speed of sound (but not below it) this expansion caused a further increase in the speed of the jet and led to a very efficient conversion of heat energy to motion. The theory of air resistance was first proposed by Sir Isaac Newton in 1726. According to him, an aerodynamic force depends on the density and velocity of the fluid, and the shape and the size of the displacing object. Newton's theory was soon followed by other theoretical solution of fluid motion problems. All these were restricted to flow under idealized conditions, i.e. air was assumed to posses constant density and to move in response to pressure and inertia. Nowadays steam turbines are the preferred power source of electric power stations and large ships, although they usually have a different design-to make best use of the fast steam jet, de Laval's turbine had to run at an impractically high speed. But for rockets the de Laval nozzle was just what was needed.

Computational Fluid Dynamics (CFD) is an engineering tool that assists experimentation. Its scope is not limited to fluid dynamics; CFD could be applied to any process which involves transport phenomena with it. To solve an engineering problem we can make use of various methods like the analytical method, experimental methods using prototypes. The analytical method is very complicated and difficult. The experimental methods are very costly. If any errors in the design were detected during the prototype testing, another prototype is to be made clarifying all the errors and again tested. This is a time-consuming as well as a cost-consuming process. The introduction of Computational Fluid Dynamics has overcome this difficulty as well as revolutionised the field of engineering. In CFD a problem is simulated in software and the transport equations associated with the problem is mathematically solved with computer assistance. Thus we would be able to predict the results of a problem before experimentation. The current work aims at determining an optimum divergent angle for the nozzle which would give the maximum outlet velocity and meet the thrust requirements. Flow instabilities might be created inside the nozzle due to the formation if shocks which reduce the exit mach number as well as thrust of the engine. This could be eliminated by varying the divergent angle. Here analysis has been conducted on nozzles with divergent angles $5^{\circ}, 10^{\circ}, 15^{\circ}$. Experimentation using the prototypes of each divergent angle is a costly as well as a time consuming process. CFD proves to be an efficient tool to overcome these limitations. Here in this work the trend of various flow parameters are also analysed. 
Flow Analysis and Optimization of Supersonic Rocket Engine Nozzle at Various Divergent ....

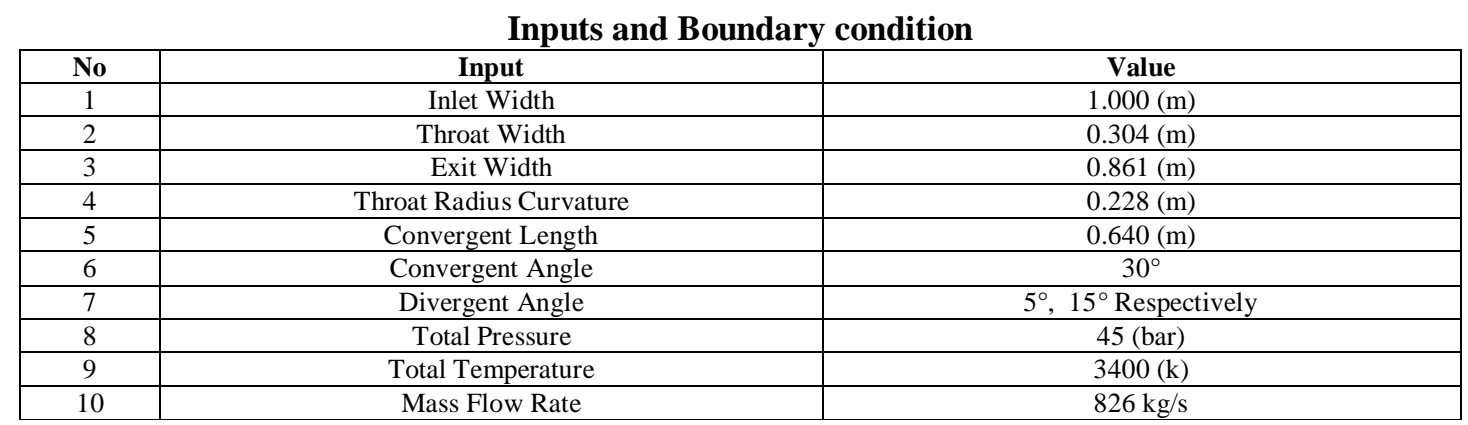

\section{Table 1: operating parameters}

\section{CFD analysis process}

\begin{tabular}{|l|c|c|}
\hline No. & Steps & Process \\
\hline 1 & Problem statement & Information about the flow and working parameters \\
\hline 2 & Mathematical model & Generate nozzle geometry \\
\hline 3 & Mesh generation & Nodes/cells, time instants \\
\hline 4 & Space discretization & Coupled ODE/DAE systems \\
\hline 5 & Time discretization & Algebraic system Ax=b \\
\hline 6 & Iterative solver & Discrete function values \\
\hline 7 & CFD software & Implementation, debugging \\
\hline 8 & Simulation run & Parameters, stopping criteria \\
\hline 9 & Post processing & Visualization, analysis of data \\
\hline 10 & Verification & Model validation / adjustment \\
\hline 11 & Saving case and data & Save all the obtain data \\
\hline & Comparing & Comparing the outcome values with real practical values \\
\hline
\end{tabular}

Table 2: General procedure for CFD analysis

\section{Meshing Pattern}

Mesh generation also called as a 'grid generation' is an important part of CFD. Meshing for CFD, is pivotal in achieving realistic renderings and physical simulations. Realistic rendering and high precision simulations depend on the quality of meshing for CFD.

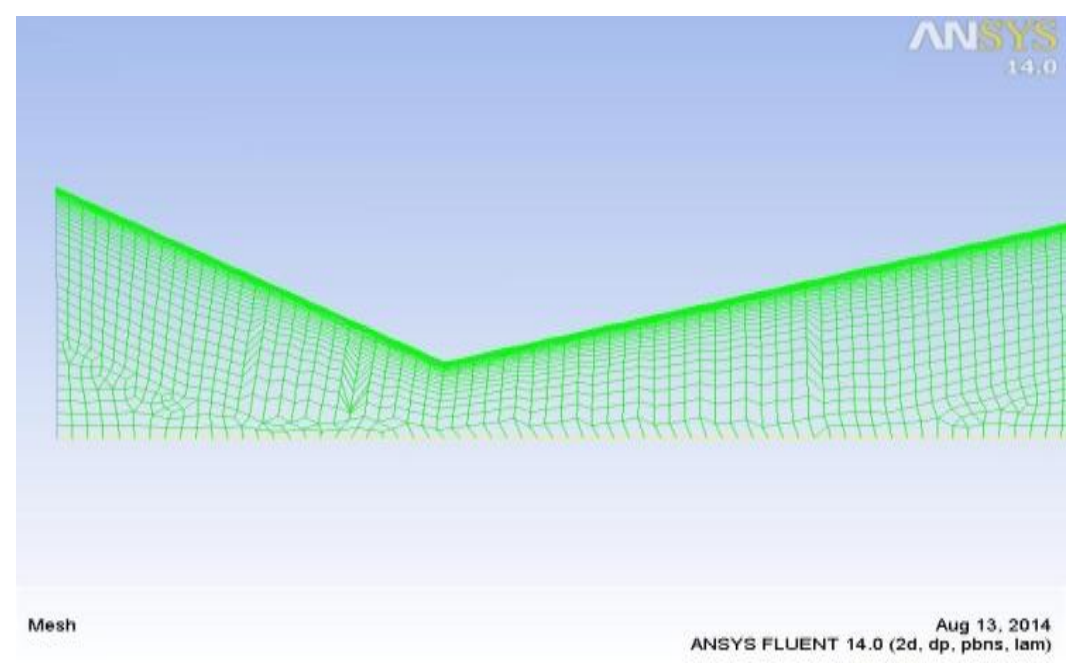

Figure 1: Mesh generation

\section{Velocity magnitude at divergent angle of $5^{\circ}$}

It can be infer from velocity contour of the nozzle that the divergent angle of $5^{\circ}$ shows the formation of oblique shock in the divergent section. As it is infer in Figure 3, Across the shock, the velocity suddenly drops from $1.38 \mathrm{e}+03 \mathrm{~m} / \mathrm{s}$ to $1.18 \mathrm{e}+03 \mathrm{~m} / \mathrm{s}$. After this the velocity of flow again increases. The positions where the shock occurs can be determined from the Velocity magnitude Vs position plot. 


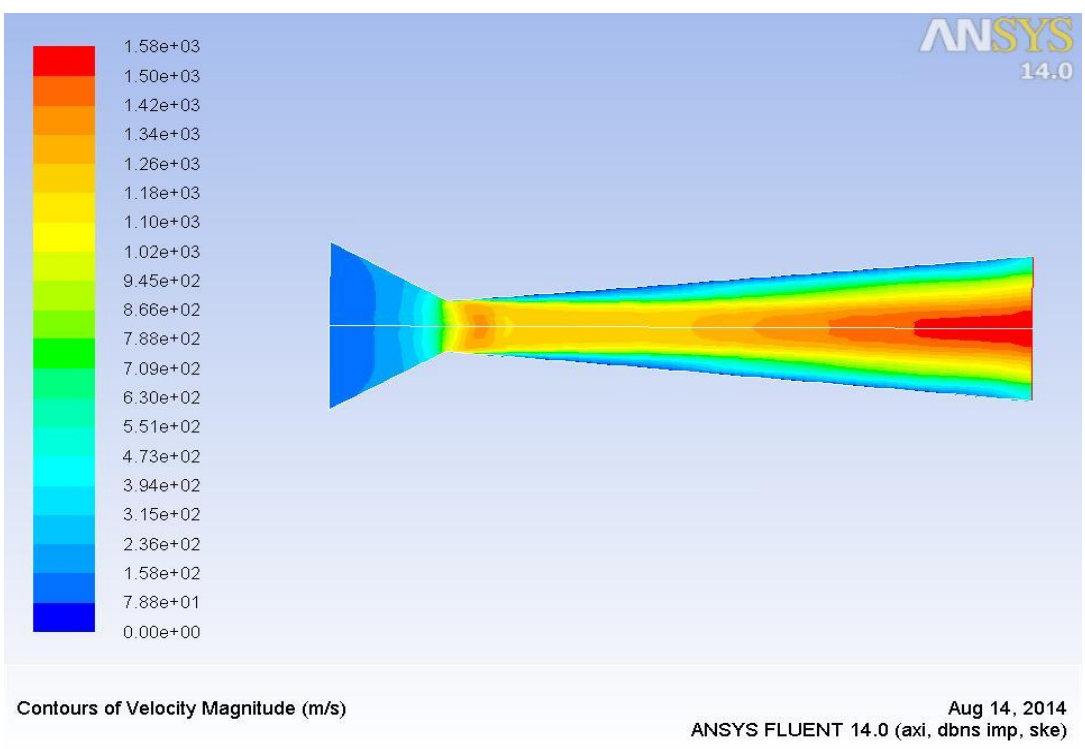

Figure 2: Contour of velocity (Mach no.) at divergent angle of $5^{\circ}$

You can easily visualize effect of viscous near the wall of nozzle which resulted in to lower velocity region depicted by string of blue shade. It is found that shock occurs at the position $0.25 \mathrm{~m}$ from the throat section. The velocity magnitude is found to increase as we move from inlet to exit. The velocity at the inlet is $0.98 \mathrm{e}+02 \mathrm{~m} / \mathrm{s}$ (sub-sonic). At the throat section the velocity varies from $4.32 \mathrm{e}+02 \mathrm{~m} / \mathrm{s}$ to $1.35 \mathrm{e}+03 \mathrm{~m} / \mathrm{s}$. The velocity at the exit was found to be $1.592 \mathrm{e}+03 \mathrm{~m} / \mathrm{s}$ (super-sonic). From this plot it is clearly observed that the velocity is increased as goes from inlet to outlet section of nozzle.

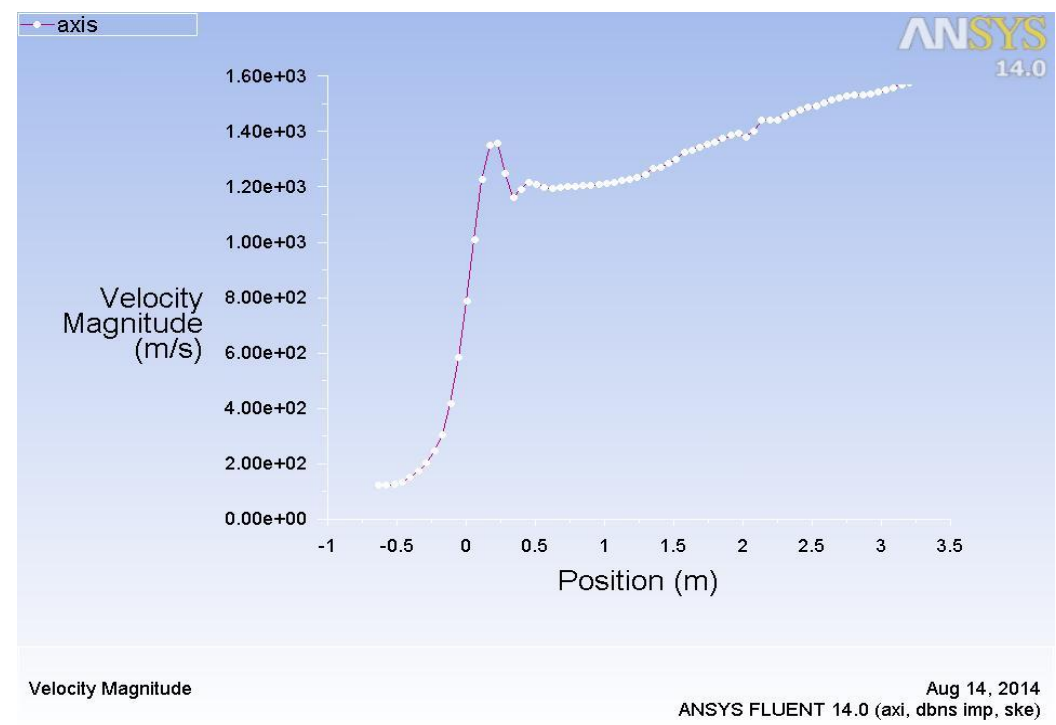

Figure 3: Velocity magnitude VS Position plot at $5 \circ$ of divergent angle

\section{Static Pressure at divergent angle of $5^{\circ}$}

Static pressure is the pressure that is exerted by a fluid. Specifically, it is the pressure measured when the fluid is still, or at rest. Contours of static pressure in convergent, throat, divergent and exit section is shown. The below figure reveals the fact that the gas gets expanded in the nozzle exit. The static pressure in the inlet is observed to be $8.52 \mathrm{e}+06 \mathrm{~Pa}$ and as we move towards the throat there is a decrease and the value at the throat is found out to be $5.88 \mathrm{e}+06 \mathrm{~Pa}$. After the throat, there is a sudden increase in the static pressure at the axis which indicates the occurrence of the shock. Then it reduces to a value of $1.36 \mathrm{e}+05 \mathrm{~Pa}$ at the exit section due to the expansion of the fluid towards the exit of the nozzle. As we can see that, maximum static pressure was noted at combustion chamber. 


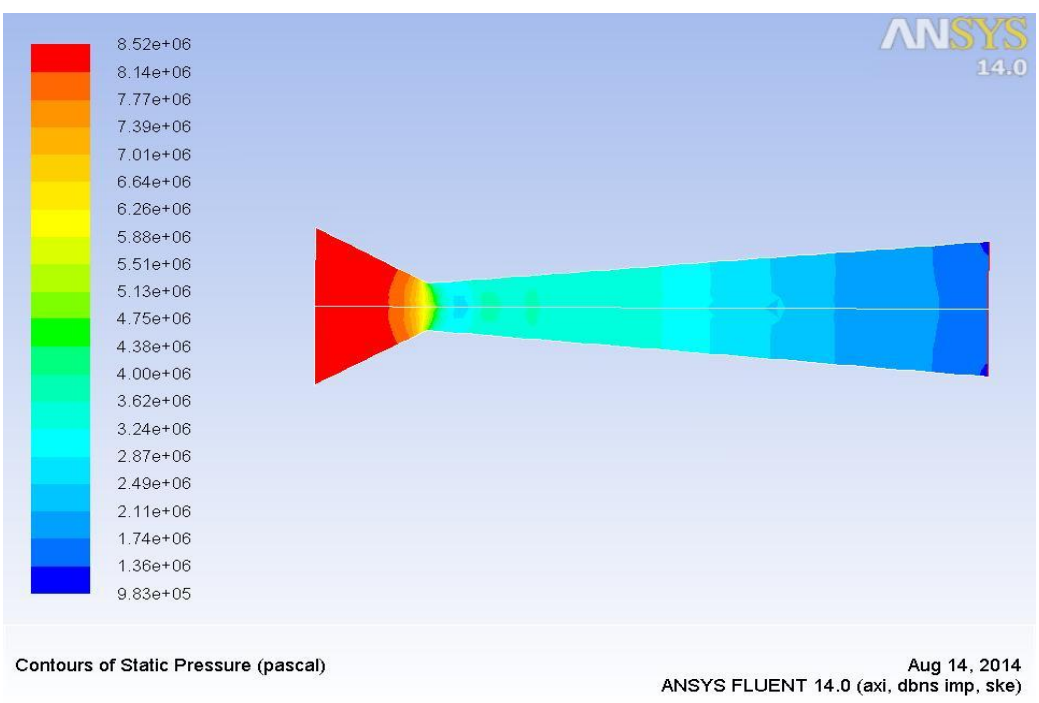

Figure 4: Contour of static pressure at divergent angle of $5^{\circ}$

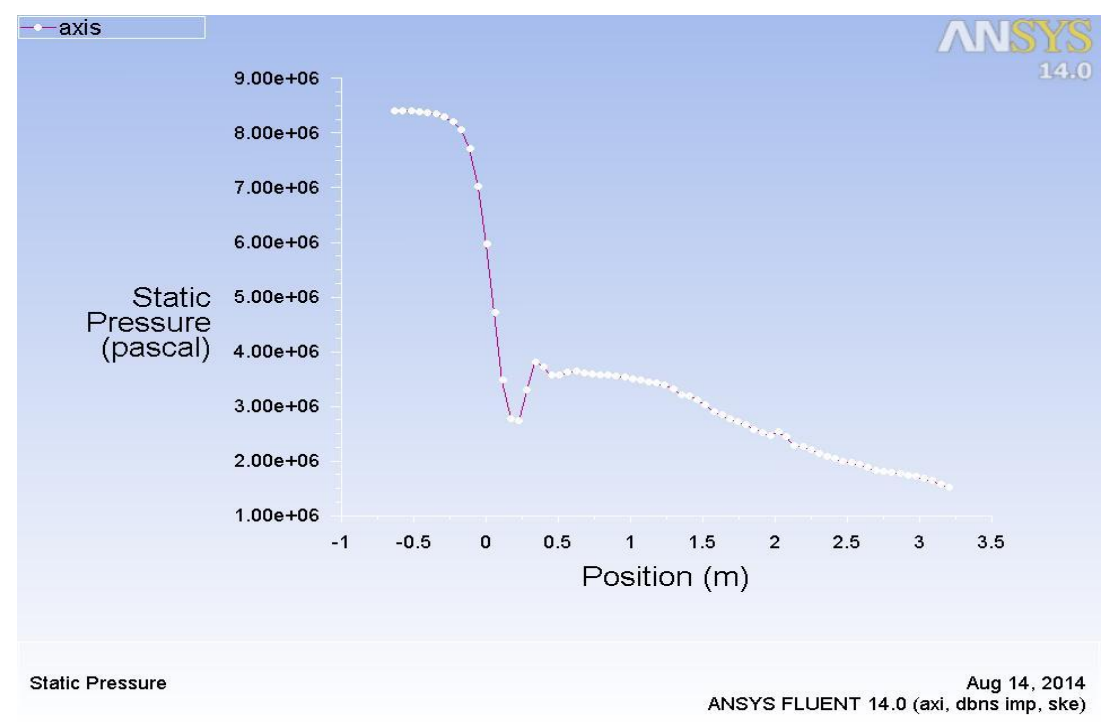

Figure 5: Static Pressure VS Position plot at $5^{\circ}$ of divergent angle

\section{Static Temperature at divergent angle of $5^{\circ}$}

The temperature almost remains a constant from the inlet up to the throat after which it tends to decrease. At the inlet and the throat the temperature is $3.32 \mathrm{e}+03 \mathrm{~K}$. After the throat, the temperature decreases till the exit. As we move from the centre vertically upwards and downward temperature increased. As we have assumed the combustion property of fluid, the static pressure is directly proportional to the static temperature. The static temperature decrease corresponding to decrease in static pressure. There is formation of shock hence static temperature increase due to decrease mach number across the shock. Static temperature is higher in combustion chamber compared to throat and divergent. 


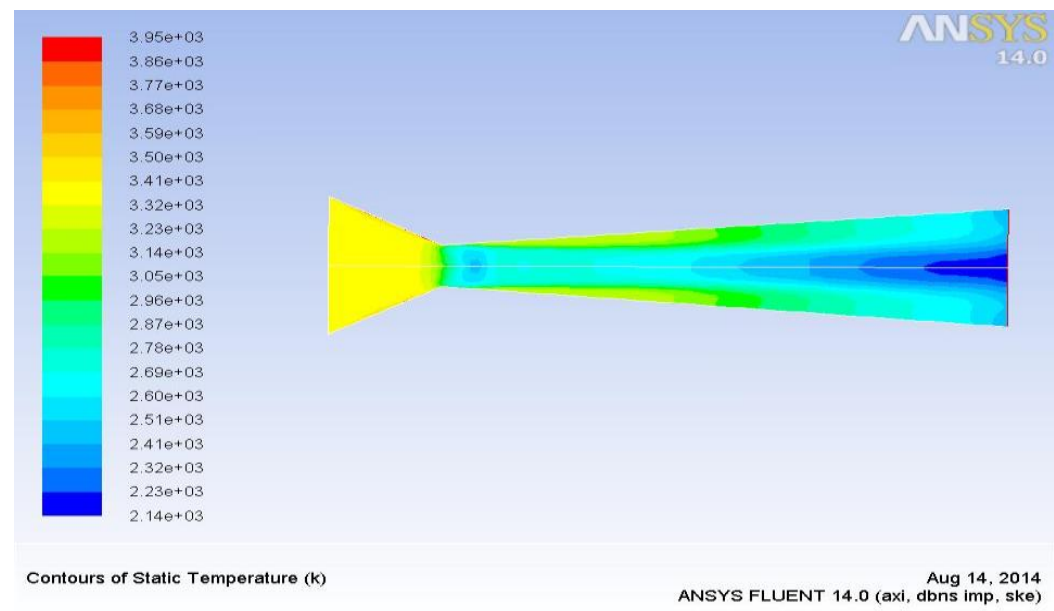

Figure 6: Contour of Static Temperature at $5^{\circ}$ of divergent angle

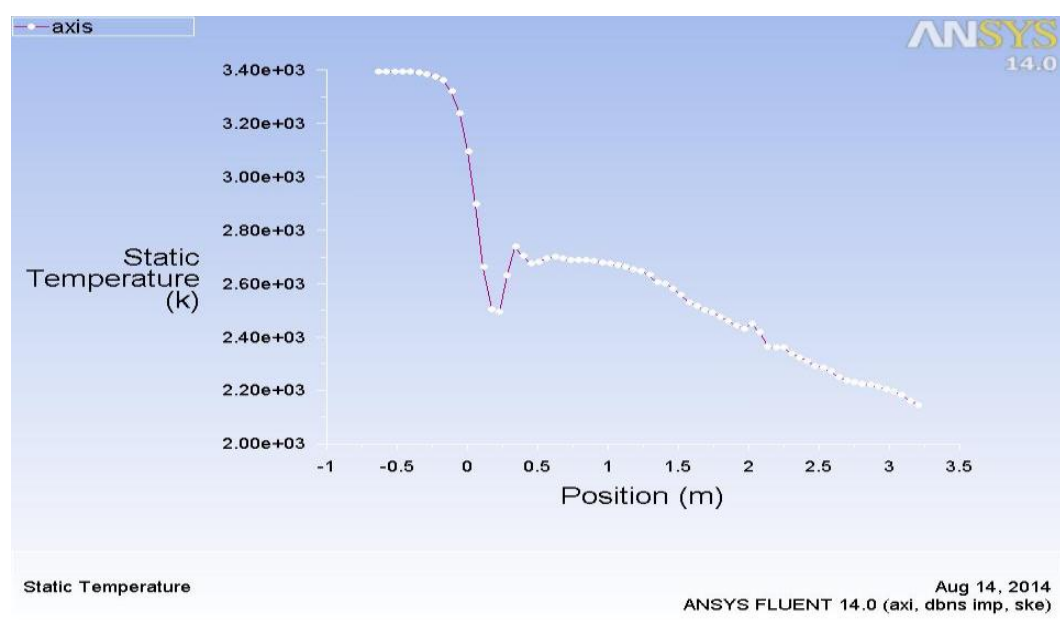

Figure 7: Static Temperature VS Position plot at $5^{\circ}$ of divergent angle

\section{Turbulent intensity at divergent angle of $5^{\circ}$}

The turbulent intensity contour shows that the inlet section has very low turbulence of the value $1.01 \mathrm{e}+00 \%$ and it increases towards the nozzle. Just as the divergent section starts the contour show very high value of turbulent intensity which is due to the sudden expansion of the flow into the divergent section. Here in this case the flow in the divergent section is highly turbulent because of the formation of two shocks inside the section. From the contour the region of shock has a turbulence intensity of $2.24 \mathrm{e}+01 \%$ and then it drops to $1.56 \mathrm{e}+01 \%$ at the exit section.

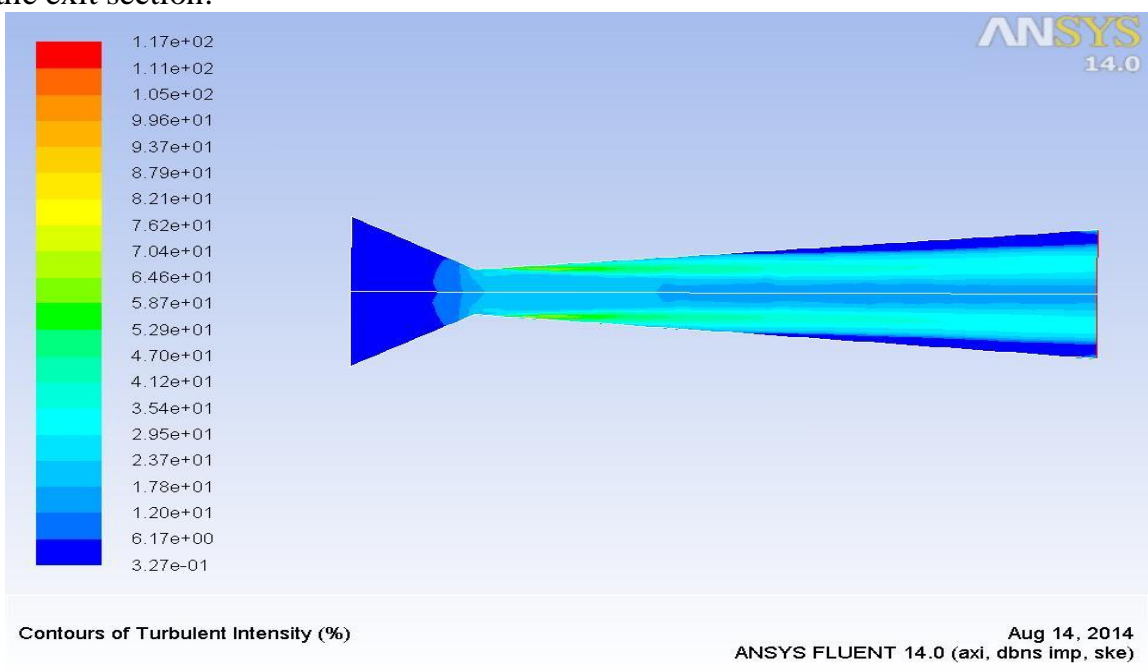

Figure 8: Contour of turbulent intensity at divergent angle of $5^{\circ}$ 


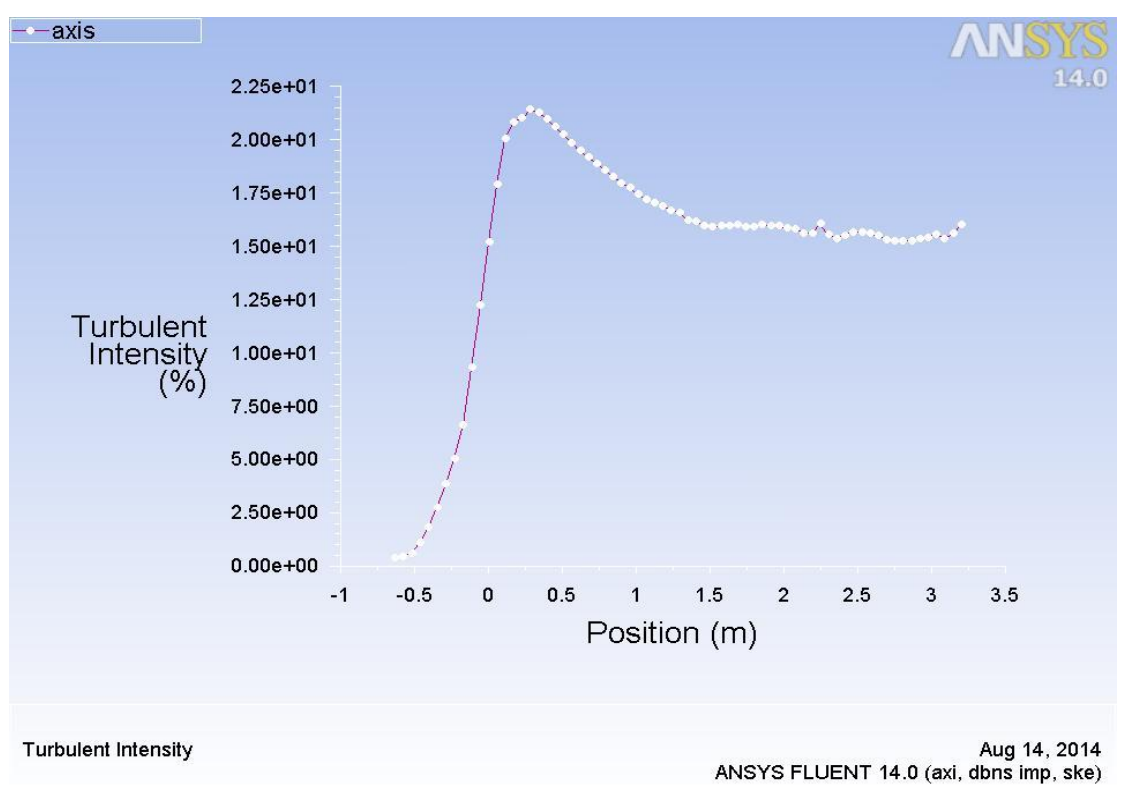

Figure 9: Turbulent intensity VS Position plot at $5^{\circ}$ of divergent angle

\section{Velocity magnitude at divergent angle of $15^{\circ}$}

The contour of velocity magnitude of convergent-divergent nozzle when the divergent angle is made $15^{\circ}$ is shown in the figure 10. It is clear that the shock has been completely eliminated from the divergent section of the nozzle. The inlet section has a velocity of $1.11 \mathrm{e}+03 \mathrm{~m} / \mathrm{s}$ and it increases to a value of $3.61 \mathrm{e}+01 \mathrm{~m} / \mathrm{s}$ at the throat section. The velocity is found to be increasing as it passes through the divergent section. At the exit section, the velocity is found to be $5.28 \mathrm{e}+03 \mathrm{~m} / \mathrm{s}$. Parallel flow is observed which is a characteristic of the conical nozzle and its design purpose (for supersonic speed) is also solved. Velocity magnitude near the wall is less due to the viscosity and the turbulence. The velocity magnitude for the default 5 degrees angle turns out to be $1.592 \mathrm{e}+02 \mathrm{~m} / \mathrm{s}$ but for a divergence angle of 20 degrees it comes out to be $5.28 \mathrm{e}+03 \mathrm{~m} / \mathrm{s}$. This is due to the change in the geometry hence flow pattern also change.

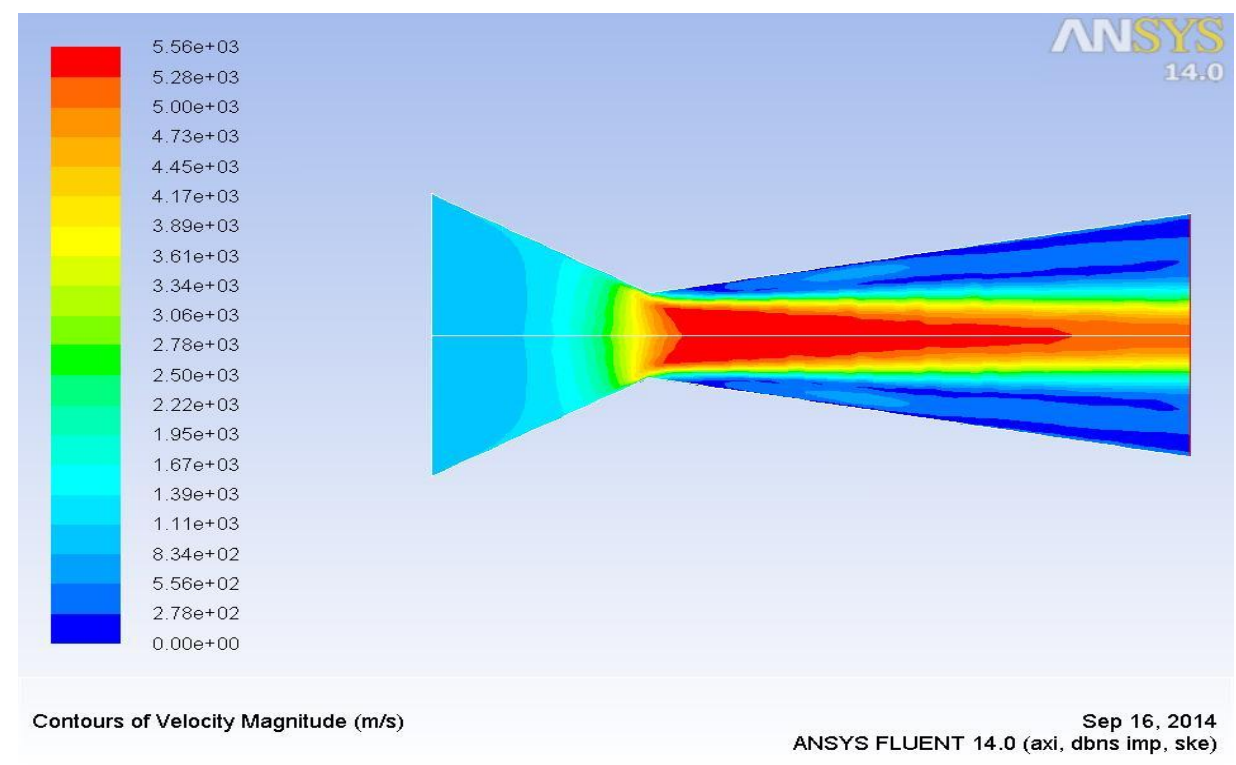

Figure 10: Contour of Velocity magnitude at divergent angle of $15^{\circ}$ 


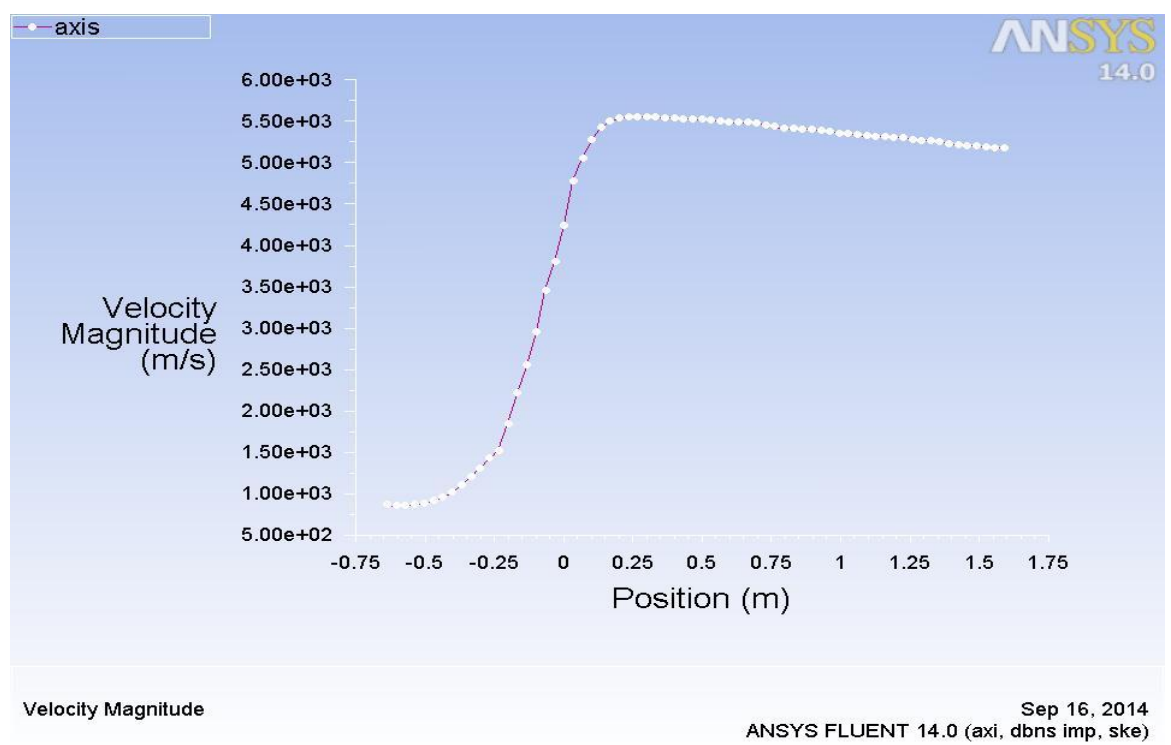

\section{Figure 11: Velocity magnitude VS Position plot at $15^{\circ}$ divergent angle}

\section{Static Pressure at divergent angle of $15^{\circ}$}

The below Figure 12, shows that the gas gets over expanded in the nozzle exit and oblique shock is prevented. The static pressure in the inlet is observed to be $1.80 \mathrm{e}+07 \mathrm{~Pa}$ and the value at the throat is found out to be $5.40 \mathrm{e}+06 \mathrm{~Pa}$. At the exit the pressure is experienced to be $1.25 \mathrm{e}+05 \mathrm{~Pa}$. Right from the inlet to the throat to the exit the static pressure tends to decrease and remains constant till exit section. There is a considerable decrease observed after the throat to the exit where there is a large drop in the static pressure. As compared to the previous case of divergent angle of 15 degree there is a change in the exit static pressure value because of change in a geometry of nozzle. This reduction in pressure intensity is directly proportional to increment in kinetic energy in form of thrust gained from nozzle at outlet section, an essential property for successful launching of rocket.

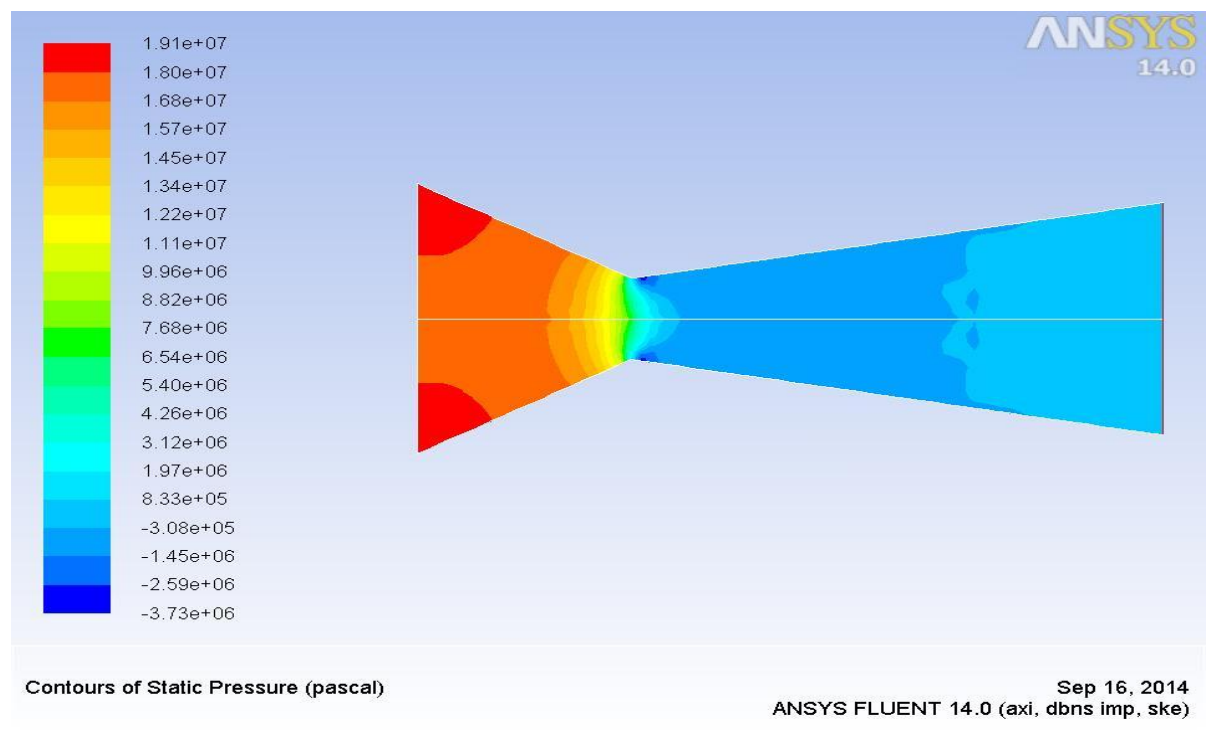

Figure 12: Contour of Static Pressure at divergent angle of $15^{\circ}$ 


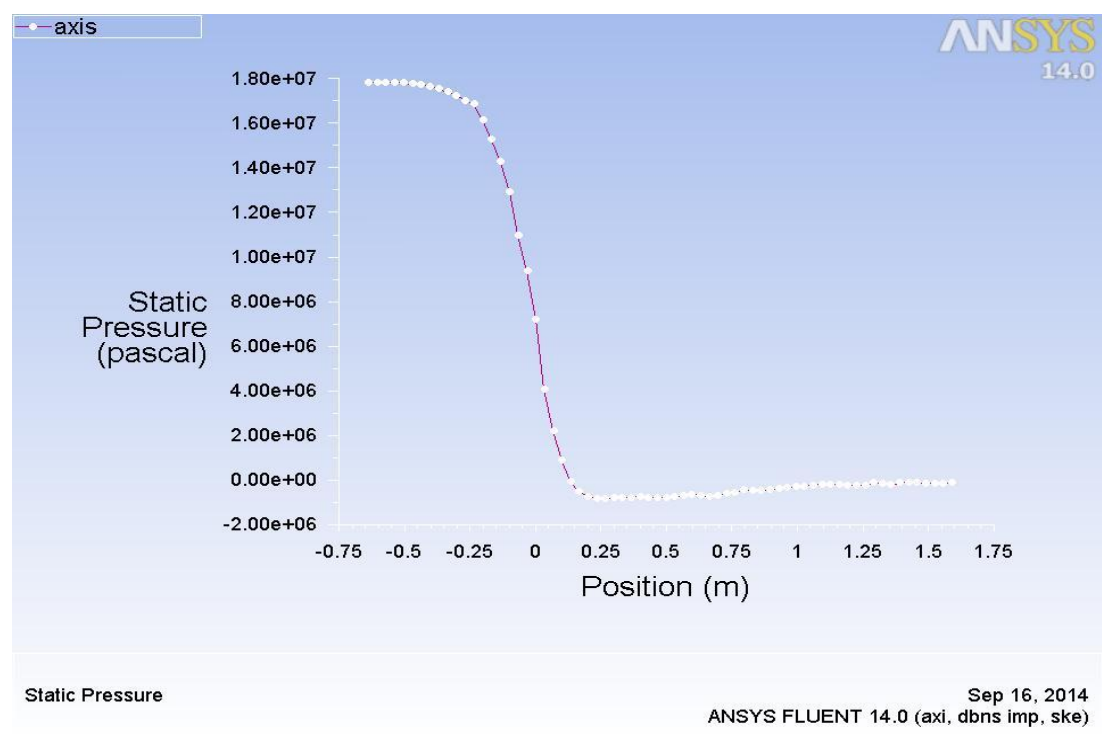

Figure 13: Static Pressure VS Position plot at $15^{\circ}$ of divergent angle

\section{Static Temperature at divergent angle of $15^{\circ}$}

The static temperature almost remains a constant in the inlet up to the throat. Increase is observed after some distance from the throat towards the exit as seen in the below Figure:14, Near the walls the temperature decreases to $2.65 \mathrm{e}+03 \mathrm{~K}$. In the inlet and the throat the temperature is $3.32 \mathrm{e}+03 \mathrm{~K}$. After the throat, the temperature increases to $4.06 \mathrm{e}+03 \mathrm{~K}$ at the exit. At the exit, moving vertically upward there is variation. The maximum value is not attained at the centre but at some distance from the centre. At the centre it is $3.59 \mathrm{e}+03 \mathrm{~K}$ and at the wall it is $2.65 \mathrm{e}+03 \mathrm{~K}$. The maximum value of $4.06 \mathrm{e}+03 \mathrm{~K}$ is attained near the walls but some distance away from it. As pressure energy of combustion gases convert in to high kinetic energy in order to generate thrust, this increment in kinetic energy also increase collision between molecules and static temperature goes up, while total temperature remains constant. The fact that in the default nozzle with 5 degrees of divergent angle, the temperature increases as soon as the throat is crossed but in this it is clearly observed that there is a beginning in the rise in temperature after some distance from the throat.

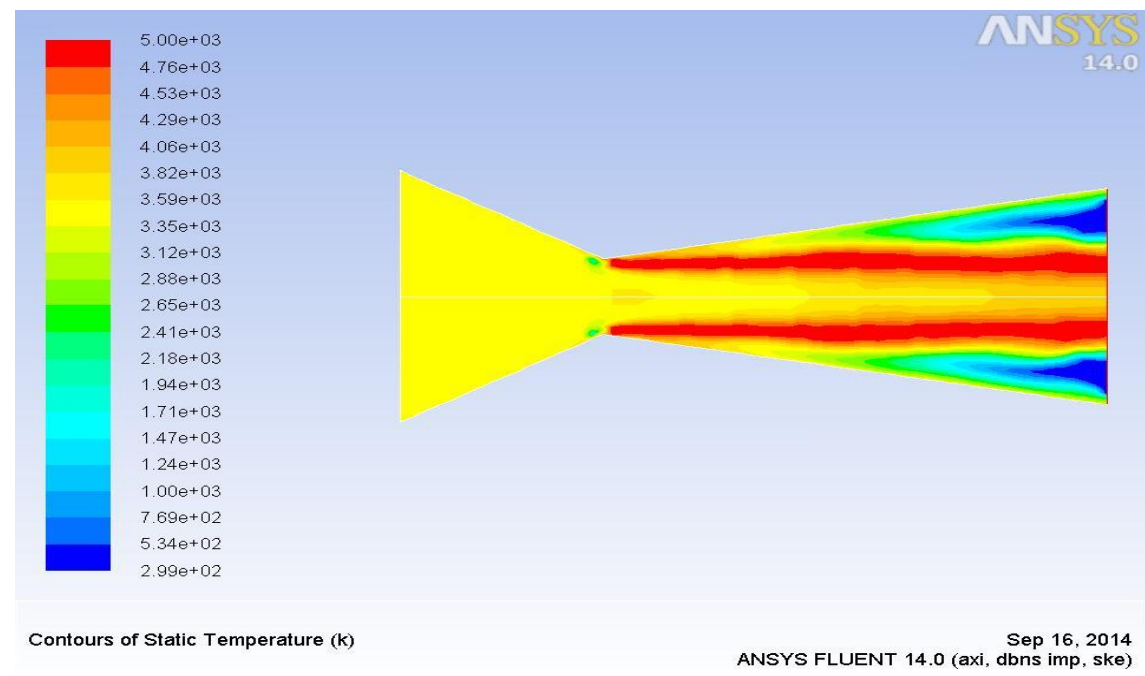

Figure 14: Contour of Static Temperature at divergent angle of $15^{\circ}$ 


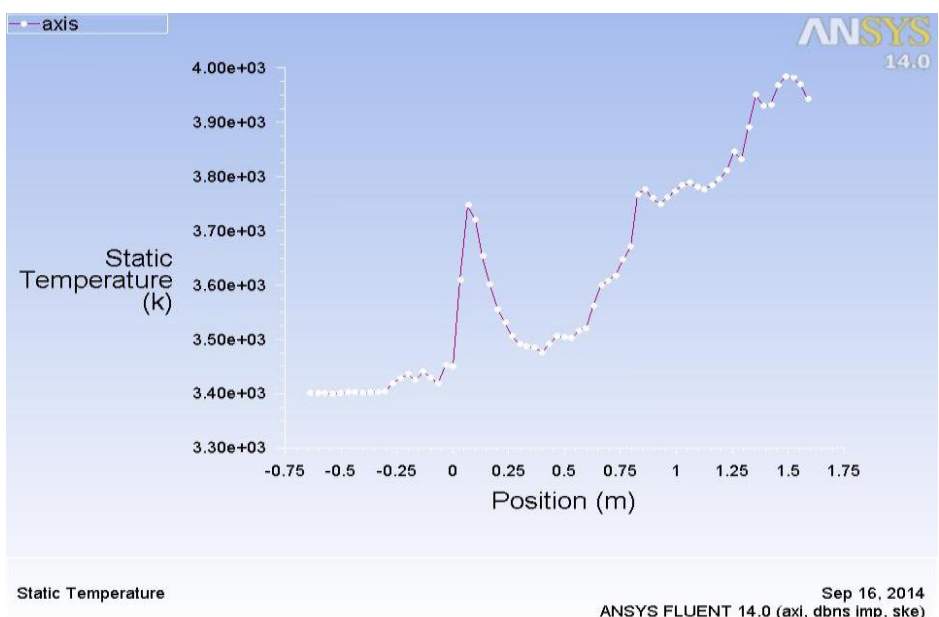

\section{Figure 15: Static Temperature VS Position plot at $15^{\circ}$ divergent angle}

\section{Turbulent intensity at divergent angle of $15^{\circ}$}

The turbulent intensity contour shows that at the inlet the turbulent intensity is $1.01 \mathrm{e}+00 \%$. It has increased to $2.40 \mathrm{e}+01 \%$ at the throat section. Since the divergent angle is higher, the sudden expansion has caused the turbulence at the beginning of the divergent section. It is also seen that the increasing velocity towards the exit section also has caused a turbulence of flow towards the exit section. The value of turbulence is found to be $2.25 \mathrm{e}+01 \%$ in this region.

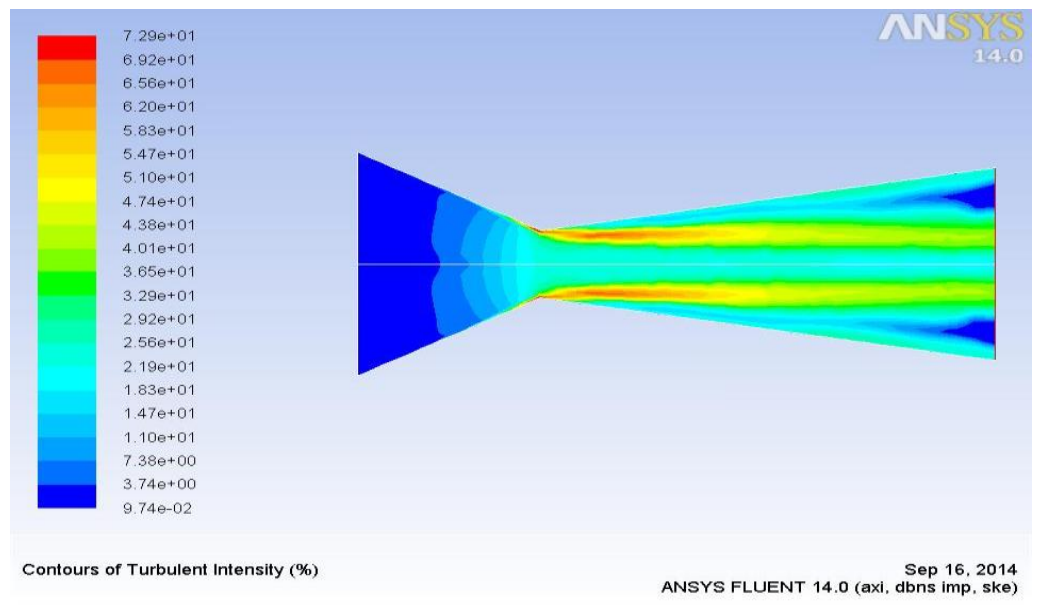

Figure 16: Turbulent intensity VS Position plot at $15^{\circ}$ divergent angle

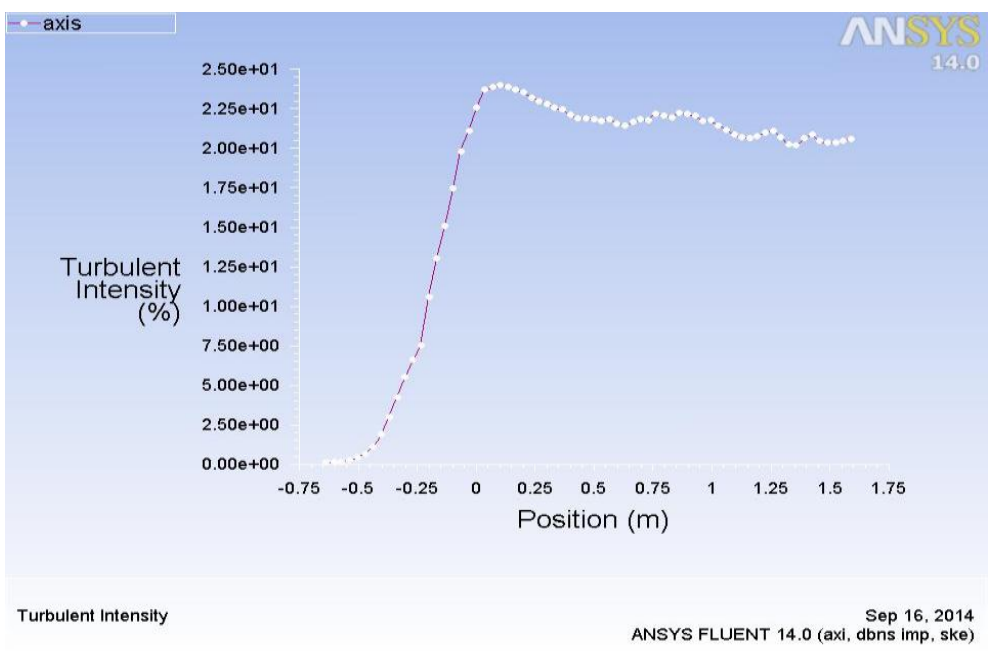

Figure 17: Turbulent intensity VS Position plot at $15^{\circ}$ divergent angle 
Flow Analysis and Optimization of Supersonic Rocket Engine Nozzle at Various Divergent ....

\section{Conclusion}

Influence of variation in divergent angle on different flow property is shown in below two tables.

Properties at throat section

\begin{tabular}{|c|c|c|c|c|c|}
\hline $\begin{array}{c}\text { No } \\
\cdot\end{array}$ & $\begin{array}{c}\text { Divergent } \\
\text { angle } \\
(\text { degrees })\end{array}$ & $\begin{array}{c}\text { Velocity } \\
\text { magnitude } \\
(\mathrm{m} / \mathbf{s})\end{array}$ & $\begin{array}{c}\text { Static } \\
\text { pressure } \\
(\text { Pascal })\end{array}$ & $\begin{array}{c}\text { Turbulent } \\
\text { intensity } \\
(\%)\end{array}$ & $\begin{array}{c}\text { Static } \\
\text { Temp. } \\
(\mathbf{K})\end{array}$ \\
\hline 1 & 5 & $1.35 e+03$ & $5.88 e+06$ & $2.24 e+01$ & $3.32 e+03$ \\
\hline 2 & 10 & $2.5 e+03$ & $5.62 e+06$ & $2.30 e+01$ & $3.25 e+03$ \\
\hline 3 & 15 & $3.61+01$ & $5.40 e+06$ & $2.40 e+01$ & $3.02 e+03$ \\
\hline
\end{tabular}

Table 1: values at throat section Properties at exit section

\begin{tabular}{|c|c|c|c|c|c|}
\hline No. & $\begin{array}{c}\text { Divergent } \\
\text { angle } \\
\text { (degrees) }\end{array}$ & $\begin{array}{c}\text { Velocity } \\
\text { magnitude } \\
(\mathbf{m} / \mathbf{s})\end{array}$ & $\begin{array}{c}\text { Static } \\
\text { pressure } \\
(\text { Pascal })\end{array}$ & $\begin{array}{c}\text { Turbulent } \\
\text { intensity } \\
(\%)\end{array}$ & $\begin{array}{c}\text { Static } \\
\text { Temp. }(\mathbf{K})\end{array}$ \\
\hline 1 & 5 & $1.592 \mathrm{e}+03$ & $1.36 \mathrm{e}+05$ & $1.56 \mathrm{e}+01$ & $\mathbf{2 . 1 8 e + 0 3}$ \\
\hline 2 & 10 & $3.06 e+03$ & $1.20 \mathrm{e}+05$ & $2.01 \mathrm{e}+01$ & $2.96 \mathrm{e}+03$ \\
\hline 3 & 15 & $5.28 \mathrm{e}+03$ & $1.26 \mathrm{e}+05$ & $2.25 \mathrm{e}+01$ & $4.06 \mathrm{e}+03$ \\
\hline
\end{tabular}

Table 2: Values at exit section

Above results were found after conducting this investigation. It was observed that oblique shocks are formed during flow through the nozzle, when the divergent angle was $5^{\circ}$. It is observed that the shock is completely eliminated from the nozzle when divergent angle increase to $15^{\circ}$ and this could be considered as a good design for the nozzle. At the exit section velocity magnitude is found to be increase with increment in divergent angle. similarly, at throat section velocity magnitude goes on when divergent angle increased. The static pressure decrease with increasing divergent angle. The efficiency of supersonic rock engine nozzle increase as we increases divergent angle of nozzle up to certain limit.

\section{References}

[1]. H.K.Versteeg and W.Malala Sekhara, "An introduction to Computational fluid Dynamics", British Library cataloguing pub, 4th edition, 1996.

[2]. Lars Davidson, “An introduction to turbulenceModels", Department of thermo and fluid dynamics, Chalmers university of technology, Goteborg, Sweden, November, 2003

[3]. Karna s. Patel, "CFD analysis of an aerofoil", International Journal of engineering research.

[4]. K.M. Pandey, Member IACSIT and A.P. Singh "CFD Analysis of Conical Nozzle for Mach 3 at Various Angles of Divergence with Fluent Software" 